\title{
INVARIANTS OF INTERSECTION OF CERTAIN PAIRS OF SPACE CURVES
}

\section{CHUAN-CHIH HSIUNG}

Introduction. Projective invariants of certain pairs of curves and surfaces have been derived and characterized geometrically by the author and others. In this paper we shall continue these investigations by studying in ordinary space some pairs of curves intersecting at a nonsingular point with distinct tangents. For each pair of such curves we can determine an independent projective invariant by the use of neighborhoods up to and including the third order of the curves at the common point. Within these neighborhoods of the curves at the point there is no other affine invariant which is not a projective invariant. ${ }^{1}$

\section{TWO CURVES INTERSECTING AT AN ORDINARY POINT WITH DISTINCT TANGENTS AND OSCULATING PLANES}

1. Derivation of an invariant $I$. Let two curves $C, \bar{C}$ in ordinary space intersect at a nonsingular point 0 with distinct tangents $t, \bar{t}$ and osculating planes $\tau, \bar{\tau}$ respectively. We shall first confine our attention to the case in which the line $p$ of intersection of the osculating planes $\tau, \bar{\tau}$ coincides with neither $t$ nor $\bar{t}$. Let $x, y, z$ represent the nonhomogeneous coordinates obtained from the projective homogeneous coordinates $x_{1}, \cdots, x_{4}$ by means of the relations

$$
x=x_{2} / x_{1}, \quad y=x_{3} / x_{1}, \quad z=x_{4} / x_{1} .
$$

If we choose the point 0 to be the origin, the tangents $t, \vec{t}$ and the line $p$ to be respectively the $x-, y$-, and $z$-axes, then the power series expansions of the curves $C, \bar{C}$ in the neighborhood of the point 0 may be written in the form

$$
\begin{array}{lll}
C: y=r x^{3}+\cdots, & z=a x^{2}+\cdots ; \\
\bar{C}: \quad x=\rho y^{3}+\cdots, & z=\alpha y^{2}+\cdots .
\end{array}
$$

The most general projective transformation which leaves the tangents $t, \bar{t}$ and the line $p$ unchanged is expressed in terms of the nonhomogeneous coordinates by the equations

Received by the editors April 26, 1948.

${ }^{1}$ For some pairs of plane curves and surfaces in ordinary space L. A. Santaló has recently found some affine invariants which are not projective invariants. See his paper, Affine invariants of certain pairs of curves and surfaces, Duke Math. J. vol. 14 (1947) pp. 559-574. 


$$
x=a_{22} x^{*} / D, \quad y=a_{33} y^{*} / D, \quad z=a_{44} z^{*} / D,
$$

where

$$
D \equiv 1+a_{12} x^{*}+a_{13} y^{*}+a_{14} z^{*}
$$

and the $a$ 's are arbitrary. The effect of this transformation on equations (1), (2) is to produce two other pairs of equations of the same form whose coefficients, indicated by stars, are given by the formulas

$$
\begin{array}{ll}
a_{33} r^{*}=a_{22}^{3} r, & a_{44} a^{*}=a_{22}^{2} a, \\
a_{22} \rho^{*}=a_{33}^{3} \rho, & a_{44} \alpha^{*}=a_{33}^{2} \alpha .
\end{array}
$$

By eliminating the $a$ 's from equation (5) it is easily seen that the expression

$$
I=r \alpha^{2} / \rho a^{2}
$$

is a projective invariant determined by the neighborhoods up to and including the third order of the two curves $C, \bar{C}$ at the point 0.

In view of the manner in which the $a$ 's appear in equations (5), it is evident that all other projective invariants depending on such neighborhoods of the two curves $C, \bar{C}$ at the point 0 are expressible in terms of the invariant $I$.

2. Metric and projective characterizations of the invariant $I$. For the purpose of giving a simple metric characterization of the invariant $I$, we make a projective transformation which leaves the point 0 unchanged and carries the $x-, y$-, and $z$-axes into three mutually perpendicular new axes. Let $\Gamma, \bar{\Gamma}$ be the transformed curves of $C, \bar{C}$, and $x^{\prime}, y^{\prime}, z^{\prime}$ be the nonhomogeneous Cartesian coordinates of a point in space referred to the new orthogonal coordinate system; then the power series expansions of the curves $\Gamma, \bar{\Gamma}$ in the neighborhood of the point 0 may be written in the form

$$
\begin{array}{lll}
\Gamma: & y^{\prime}=r^{\prime} x^{\prime 3}+\cdots, & z^{\prime}=a^{\prime} x^{\prime 2}+\cdots, \\
\bar{\Gamma}: & x^{\prime}=\rho^{\prime} y^{\prime 3}+\cdots, & z^{\prime}=\alpha^{\prime} y^{\prime 2}+\cdots,
\end{array}
$$

and the invariant $I$ takes the form

$$
I=r^{\prime} \alpha^{2} / \rho^{\prime} a^{\prime 2} \text {. }
$$

Let $R^{\prime}, \bar{R}^{\prime}$, and $T^{\prime}, \bar{T}^{\prime}$ be respectively the radii of curvature and of torsion of the curves $\Gamma, \bar{\Gamma}$ at the point 0 , then

$$
\begin{array}{ll}
R^{\prime}=1 / 2 a^{\prime}, & T^{\prime}=a^{\prime} / 3 r^{\prime}, \\
\bar{R}^{\prime}=1 / 2 \alpha^{\prime}, & \bar{T}^{\prime}=-\alpha^{\prime} / 3 \rho^{\prime},
\end{array}
$$


and therefore

$$
I=-R^{\prime} \bar{T}^{\prime} / \bar{R}^{\prime} T^{\prime}
$$

Thus we obtain the following metric characterization of the invariant $I$ associated with the point 0 of intersection of the two curves $C$, $\bar{C}$.

Let $R, \bar{R}$, and $T, \bar{T}$ be respectively the radii of curvature and of torsion of the curves $C, \bar{C}$ at the point 0 , then

$$
I=-R \bar{T} / \bar{R} T
$$

In order to interpret the invariant $I$ projectively we observe that among the five-point quadric cones of the curve $C$ at the point 0 we may determine a unique one which has $\bar{t}$ for a generator and whose tangent plane along $\bar{t}$ passes through the line $p$. From expansions (1) follows immediately the equation of this cone, namely,

$$
a^{2} x y-r z^{2}=0 \text {. }
$$

Similarly, with the roles of the curves $C, \bar{C}$ interchanged we have another quadric cone:

$$
\alpha^{2} x y-\rho z^{2}=0 .
$$

The two cones (13), (14) determine a pencil $P$ of quadric cones, to which belong the pair of planes $x=0, y=0$ and the plane $z=0$, counted twice. Hence the invariant $I$ is equal to the cross ratio of the cones (13), (14) and these two pairs of planes in the pencil $P$ of quadric cones.

3. Derivation of an invariant $J$. We next consider in ordinary space two curves $C, \bar{C}$ intersecting at a nonsingular point 0 with distinct tangents $t, \bar{t}$ and osculating planes $\tau, \bar{\tau}$, respectively, the line $p$ of intersection of the osculating planes $\tau, \bar{\tau}$ coinciding with the tangent $\vec{t}$. If we choose the point 0 to be the origin, the tangents $t, \bar{t}$ to be the $x$ - and $y$-axes respectively, and any line through the point 0 and in the osculating plane $\bar{\tau}$ to be the $z$-axis, then the power series expansions of the curves $C, \bar{C}$ in the neighborhood of the point 0 may be written in the form

$$
\begin{array}{lll}
C: z=r x^{3}+\cdots, & y=a x^{2}+\cdots ; \\
\bar{C}: \quad x=\rho y^{3}+\cdots, & z=\alpha y^{2}+\cdots .
\end{array}
$$

As in $\$ 1$, we make the most general projective transformation of coordinates

$$
x=a_{22} x^{*} / D, \quad y=\left(a_{33} y^{*}+a_{34} z^{*}\right) / D, \quad z=a_{44} z^{*} / D
$$


which leaves the tangents $t, \bar{t}$ and the osculating plane $\bar{\tau}$ unchanged, where $D$ is defined by expression (4) and the $a^{\prime}$ s are arbitrary. The effect of this transformation on equations (15), (16) is to produce two other pairs of equations of the same form whose coefficients, indicated by stars, are given by the formulas

$$
\begin{array}{ll}
a_{44} r^{*}=a_{22}^{3} r, & a_{33} a^{*}=a_{22}^{2} a, \\
a_{22} \rho^{*}=a_{33}^{3} \rho, & a_{44} \alpha^{*}=a_{33}^{2} \alpha .
\end{array}
$$

Eliminating the $a$ 's from equations (18) we may obtain an independent projective invariant

$$
J=r^{5} \rho / a^{7} \alpha^{5}
$$

determined by the neighborhoods up to and including the third order of the two curves $C, \bar{C}$ at the point 0 .

4. Metric and projective characterizations of the invariant $J$. Let $R, \bar{R}$ and $T, \bar{T}$ be respectively the radii of curvature and of torsion at the point 0 of the curves $C, \bar{C}$ in the preceding section, then it is easily seen that

$$
J=\left(\frac{2}{3}\right)^{6} \frac{R^{2} \bar{R}^{4}}{T^{5} \bar{T}} .
$$

Among the five-point quadric cones of the curve $C$ at the point 0 , we may determine a unique one which has the $z$-axis as a generator and whose tangent plane along the $z$-axis is the plane $x=0$. The equation of this cone is easily found, from expansions (15), to be

$$
a^{2} x z-r y^{2}=0 .
$$

Similarly, the equation of the unique five-point quadric cone of the curve $\bar{C}$ at the point 0 having the $x$-axis as a generator and the $x z$ plane as tangent plane along that axis is

$$
\alpha^{2} x y-\rho z^{2}=0 .
$$

The $z$-axis and the three lines of intersection, other than the $x$-axis, of the two quadric cones (21), (22) determine three planes through the $z$-axis, which intersect the plane $z=0$ in the three lines

$$
z=a^{4} \alpha^{2} x^{3}-r^{2} \rho y^{3}=0 .
$$

On the other hand, from expansions (15), (16) we may easily obtain the equation of any quadric which has the $x$-axis as a generator, and which has four-point contact with the curve $C$ at the point 0 
and three-point contact with the curve $\bar{C}$ at the point 0 ; the result is

$$
r x y+b_{13} x z+a \alpha y^{2}+b_{23} y z+b_{33} z^{2}-a z=0,
$$

where the $b$ 's are arbitrary. The plane $z=0$ cuts the quadric (24) in the $x$-axis and the line $u$ :

$$
z=r x+a \alpha y=0 .
$$

Let $v$ be any one of the three lines (23), then the cross ratio of the four lines $t, \bar{t}, u, v$ is equal to $-J^{1 / 3}$.

\section{TWO CURVES INTERSECTING AT AN ORDINARY POINT WITH DISTINCT TANGENTS BUT THE SAME OSCULATING PLANE}

5. An invariant and its metric and projective characterizations. Finally, we consider in ordinary space two curves $C, \bar{C}$ intersecting at a nonsingular point 0 with distinct tangents $t, \bar{t}$, respectively, but the same osculating plane $\tau$. If we choose the point 0 to be the origin, the tangents $t, \bar{t}$ to be respectively the $x$ - and $y$-axes, and any line through the point 0 but not in the common osculating plane $\tau$ to be the $z$-axis, then the power series expansions of the curves $C, \bar{C}$ in the neighborhood of the point 0 may be written in the form

$$
\begin{array}{lll}
C: z=r x^{3}+\cdots, & y=a x^{2}+\cdots ; \\
\bar{C}: z=\rho y^{3}+\cdots, & x=\alpha y^{2}+\cdots .
\end{array}
$$

In a way similar to the foregoing we can easily show that the expression

$$
K=r \alpha / a \rho
$$

is an independent projective invariant determined by the neighborhoods $u p$ to and including the third order of the two curves $C, \bar{C}$ at the point $0 .^{2}$

Let $T, \bar{T}$ be respectively the radii of torsion of the curves $C, \bar{C}$ at the point 0 , then it follows immediately that

$$
K=-\bar{T} / T \text {. }
$$

It is known ${ }^{3}$ that associated with the point 0 of the curves $C, \bar{C}$ there are three principal lines whose equations are

${ }^{2}$ For two plane curves intersecting at an ordinary point no projective invariant can be determined by the neighborhoods up to and including the third order of the curves at the common point. See the author's paper, Theory of intersection of two plane curves, Bull. Amer. Math. Soc. vol. 49 (1943) pp. 786-792.

${ }^{3} \mathrm{E}$. Bompiani, Invarianti d'intersezione di due curve sghembe, Rendiconti R. Accademia Nazionale dei Lincei (6) vol. 14 (1931) p. 459. 


$$
z=r x^{3}+\rho y^{3}=0 .
$$

On the other hand, let $Q(\bar{Q})$ be any three-point quadric of the curve $C(\bar{C})$ at the point 0 satisfying the condition that the tangent plane of $Q(\bar{Q})$ at the other point $\bar{S}(S)$ of intersection of $Q(\bar{Q})$ with the tangent $\bar{t}(t)$ passes through the point $S(\bar{S})$. Then the lines joining the point 0 to the other three points of intersection of the two conics determined by the quadrics $Q, \bar{Q}$ and the common osculating plane $\tau$ have the equations ${ }^{4}$

$$
z=a x^{3}-\alpha y^{3}=0 .
$$

If $u$ be any one of the principal lines (30) and $v$ be any one of the three lines (31), then the projective invariant $K$ associated with the point 0 of intersection of the curves $C, \bar{C}$ is, except for sign, equal to the cube of the cross ratio $(t, \bar{t}, u, v)$.

\section{Michigan State College}

${ }^{4}$ C. C. Hsiung, Note on the intersection of two space curves, Tôhoku Math. J. vol. 47 (1940) pp. 206-207. 